\title{
Universal aspects of string propagation on curved backgrounds
}

\author{
Ioannis Bakas* \\ Theory Division, CERN, 1211 Geneva 23, Switzerland \\ and Department of Physics, University of Patras, 26110 Patras, Greece ${ }^{\dagger}$ \\ Konstadinos Sfetsos \\ Institute for Theoretical Physics, Utrecht University, Princetonplein 5, TA 3508, The Netherlands \\ (Received 2 May 1996)
}

\begin{abstract}
String propagation on $D$-dimensional curved backgrounds with Lorentzian signature is formulated as a geometrical problem of embedding surfaces. When the spatial part of the background corresponds to a general WZW model for a compact group, the classical dynamics of the physical degrees of freedom is governed by the coset conformal field theory $\mathrm{SO}(D-1) / \mathrm{SO}(D-2)$, which is universal irrespective of the particular WZW model. The same holds for string propagation on $D$-dimensional flat space. The integration of the corresponding Gauss-Codazzi equations requires the introduction of (non-Abelian) parafermions in differential geometry. [S0556-2821(96)01418-X]
\end{abstract}

PACS number(s): 11.25.Sq, 11.25.Hf, 04.62.+v

\section{INTRODUCTION}

String propagation on a given background defines an embedding problem in differential geometry. Choosing, whenever possible, the temporal gauge one may solve the Virasoro constraints and consider the nonlinear dynamics governing the physical degrees of freedom of the string. Simple counting shows that for $D$-dimensional backgrounds the physical degrees of freedom satisfy a coupled system of $D-2$ differential equations, which are defined on the twodimensional string world sheet and they are nonlinear due to the quadratic form of the Virasoro constraints. Our primary aim is to investigate the integrability of these equations and explore some of their universal aspects for a wide class of backgrounds.

Lund and Regge considered this problem several years ago for string propagation on flat four-dimensional (4D) Minkowski space, in the presence of a Kalb-Ramond field as well [1]. This geometrical approach was subsequently generalized to $D \geqslant 5$ [2]. It became clear more recently [3] that the dynamics of the physical degrees of freedom in the $D=4$ case admits a Lagrangian formulation as an $\mathrm{SO}(3) / \mathrm{SO}(2)$ gauged Wess-Zumino-Witten (WZW) model. However, for $D \geqslant 5$ an analogous Lagrangian description using cosets to model the dynamics of the $D-2$ physical degrees of freedom has been lacking. The technical problem that arises here is finding the appropriate nonlocal field variables to integrate the underlying Gauss-Codazzi equations of the embedding. We solve this problem by introducing, just from purely geometrical considerations, the non-Abelian parafermions of the coset model $\mathrm{SO}(D-1) / \mathrm{SO}(D-2)$ and show that the chiral equations they obey [4] are equivalent to the Gauss-Codazzi embedding equations. Hence string dynamics on $D$-dimensional flat Minkowski space, after we solve the $\mathrm{Vi-}$

\footnotetext{
*Electronic address: BAKAS@SURYA11.CERN.CH

${ }^{\dagger}$ Permanent address.

"Electronic address: SFETSOS@FYS.RUU.NL
}

rasoro constraints, is governed by the semiclassical geometry of the conformal field theory coset $\mathrm{SO}(D-1) / \mathrm{SO}(D-2)$ $[4,5]$.

An interesting generalization of this program includes Lorentzian backgrounds of the product form $R \otimes K_{D-1}$, where $K_{D-1}$ is a WZW model for a semisimple compact group. The integration of the Gauss-Codazzi equations for these backgrounds is similar to flat space in that $\mathrm{SO}(D-1) / \mathrm{SO}(D-2)$ parafermions are also used, thus exhibiting a universal behavior irrespectively of the particular WZW model $K_{D-1}$. The coset space structure of the physical degrees of freedom of the free string is rather remarkable, leading to the world sheet integrability of the underlying nonlinear equations. Using the parafermion variables of the Gauss-Codazzi equations one may easily find chiral $W_{\infty}$ symmetries as hidden on-shell symmetries of the classical theory. Our results shed new light into the differential geometry of embedding surfaces using concepts and field variables, which so far have been natural only in conformal field theory.

The organization of this paper is as follows. In Sec. II we set up the Gauss-Codazzi equations for string propagation on $D$-dimensional curved space and determine a wide class of backgrounds that allow for their integration. We expose the universal aspects of string dynamics for Lorentzian backgrounds whose spatial part is either flat space or a WZW model based on a general compact group. In Sec. III we use the $\mathrm{SO}(D-1) / \mathrm{SO}(D-2) \mathrm{WZW}$ model to describe systematically the dynamics of the physical degrees of freedom and present explicit results for $D=4$ and $D=5$. Finally, in Sec. IV we comment on various other generalizations and the quantization of strings before or after solving the classical Virasoro constraints. Connections with reduced $\sigma$ models [6-9] and the associated systems of symmetric space sineGordon models are also discussed.

\section{STRING DYNAMICS AND EMBEDDING SURFACES}

We first review relevant parts from the theory of embedding surfaces in the context of Riemannian geometry (see for 
instance [10]). Then we consider classical string propagation on backgrounds with Lorentzian signature and we formulate the problem of determining the dynamics of the physical modes as a geometrical problem of surface embedding, after solving the Virasoro constraints in the temporal gauge. At the end we specialize to backgrounds with spatial part corresponding to flat space or WZW models based on general semisimple compact groups.

\section{Gauss-Codazzi equations: Generalities}

Consider a $D$-dimensional space $M_{D}$ with line element ( $\equiv$ fundamental quadratic form) given by

$$
d s_{D}^{2}=G_{\mu \nu}(y) d y^{\mu} d y^{\nu}, \quad \mu, \nu=1, \ldots, D .
$$

A $d$-dimensional subspace $M_{d}$ of $M_{D}$ with local coordinates $x^{i}, i=1, \ldots, d$ may be considered as an embedded surface with defining equations $y^{\mu}=y^{\mu}\left(x^{1}, \ldots, x^{d}\right)$. The line element in $M_{d}$ will be denoted by

$$
d s_{d}^{2}=g_{i j}(x) d x^{i} d x^{j}, \quad i, j=1, \ldots, d .
$$

The restriction of Eq. (2.1) in $M_{d}$ should be equivalent to Eq. (2.2). Thus we have the relation ${ }^{1}$

$$
g_{i j}(x)=G_{\mu \nu}(y) y_{, i}^{\mu} y_{, j}^{\nu} .
$$

The embedded surface is completely specified by the set of vectors $\left\{\xi_{\sigma}^{\mu}, \sigma=d+1, \ldots, D\right\}$ normal to it. These are chosen to satisfy the orthonormalization conditions

$$
G_{\mu \nu} \xi_{\sigma}^{\mu} \xi_{\tau}^{\nu}=\delta_{\sigma \tau}
$$

and by definition are also orthogonal to the tangent vectors to the surface $y_{, i}^{\mu}$

$$
G_{\mu \nu} y_{, i}^{\mu} \xi_{\sigma}^{\nu}=0 .
$$

The set of vectors $\left\{y_{, i}^{\mu}, \xi_{\sigma}^{\mu}\right\}$ satisfy the completeness relation in $M_{D}$ :

$$
g^{i j} y_{, i}^{\mu} y_{, j}^{\nu}+\xi_{\sigma}^{\mu} \xi_{\tau}^{\nu} \delta^{\sigma \tau}=G^{\mu \nu} .
$$

The dynamics of the embedded surface is determined from the evolution of the vectors $y_{, i}^{\mu}$ and $\xi_{\sigma}^{\mu}$ as functions of the variables $x^{i}$ in $M_{d}$. The corresponding equations are determined by repeated covariant differentiations of Eqs. (2.3)(2.5) and subsequent algebraic manipulations. Here we will only present the result leaving out the detailed proofs which can be found in [10]. We recall the concept of the second fundamental quadratic form with components defined as

$$
\Omega_{i j}^{\sigma}=G_{\mu \nu} \xi_{\sigma}^{\mu}\left(D_{i} D_{j} y^{\nu}+\Gamma_{\lambda \alpha}^{\nu} y_{, i}^{\lambda} y_{, j}^{\alpha}\right) .
$$

\footnotetext{
${ }^{1}$ We will use the notation $y_{, i}^{\mu} \equiv \partial y^{\mu} / \partial x^{i}$. Covariant derivatives on $M_{D}$ and on $M_{d}$ will be denoted by $D_{\mu}$ and $D_{i}$, respectively. The $y^{\mu}(x)$ 's are scalars with respect to covariant differentiation on $M_{d}$, i.e., $D_{i} y^{\mu}=y_{, i}^{\mu}$.
}

It is obvious that it is a symmetric tensor in $M_{d}$, i.e., $\Omega_{i j}^{\sigma}=\Omega_{j i}^{\sigma}$. We also define the torsion ( $\equiv$ third fundamental form) in $M_{d}$ :

$$
\mu_{i}^{\sigma \tau}=G_{\mu \nu} \xi_{\sigma}^{\mu}\left(\xi_{\tau, i}^{\nu}+\Gamma_{\lambda \alpha}^{\nu} \xi_{\tau}^{\lambda} y_{, i}^{\alpha}\right)
$$

Though not immediately obvious it can be shown that it is antisymmetric, i.e., $\mu_{i}^{\sigma \tau}+\mu_{i}^{\tau \sigma}=0$. With the above definitions the evolution equations can be written as

$$
D_{i} D_{j} y^{\mu}=\Omega_{i j}^{\sigma} \xi_{\sigma}^{\mu}-\Gamma_{\nu \lambda}^{\mu} y_{, i}^{\nu} y_{, j}^{\lambda},
$$

and

$$
\xi_{\sigma, i}^{\mu}=-\Omega_{i j}^{\sigma} g^{j k} y_{, k}^{\mu}+\mu_{i}^{\tau \sigma} \xi_{\tau}^{\mu}-\Gamma_{\lambda \alpha}^{\mu} y_{, i}^{\lambda} \xi_{\sigma}^{\alpha} .
$$

The careful reader will notice that for curves $(d=1)$ in 3D Euclidean space, the equations (2.9) and (2.10) reduce to the well known Serret-Frenet formulas.

It is a quite straightforward but tedious procedure to derive the necessary conditions for the existence of solutions to Eqs. (2.9) and (2.10). The resulting compatibility equations are given by

$$
\begin{gathered}
R_{i j k l}=R_{\mu \nu \alpha \beta} y_{, i}^{\mu} y_{, j}^{\nu} y_{, k}^{\alpha} y_{, l}^{\beta}+\Omega_{k[i}^{\tau} \Omega_{j] l}^{\tau}, \\
D_{[k} \Omega_{j] i}^{\sigma}=\mu_{[k}^{\tau \sigma} \Omega_{j] i}^{\tau}+R_{\mu \nu \alpha \beta} y_{, i}^{\mu} y_{, j}^{\alpha} y_{, k}^{\beta} \xi_{\sigma}^{\nu},
\end{gathered}
$$

and

$$
D_{[k} \mu_{j]}^{\sigma \tau}+\mu_{[j}^{\rho \sigma} \mu_{k]}^{\rho \tau}+\Omega_{l[j}^{\sigma} \Omega_{k] i}^{\tau} g^{l i}+R_{\mu \nu \alpha \beta} y_{, j}^{\mu} y_{, k}^{\nu} \xi_{\sigma}^{\alpha} \xi_{\tau}^{\beta}=0 .
$$

Equations (2.11) and (2.12) for the case of a 2D surface in 3D Euclidean space are known as the Gauss-Codazzi equations, whereas Eq. (2.13) for the case of a surface immersed in Euclidean space is known as the Ricci equation. In general, the number of unknown functions in the embedding equations of a space $M_{d}$ in $M_{D}$ exceeds the number of equations. However, the extra functions may be eliminated using the freedom to perform local transformations in the normal space to the surface that rotate $\Omega_{i}^{\sigma}$ and $\mu_{i}^{\sigma \tau}$, also using any additional information that might be in our disposal. The precise mechanism, for the cases of interest in this paper, will be considered in detail in the next subsection.

\section{String evolution in $M_{D}=R \otimes K_{D-1}$}

We consider classical propagation of closed strings on a $D$-dimensional background that is the direct product of the real line $R$ (contributing a minus in the signature matrix) and a general manifold (with Euclidean signature) $K_{D-1}$, i.e., $M_{D}=R \otimes K_{D-1}$. The corresponding target space variables are $y^{0}\left(\sigma^{+}, \sigma^{-}\right)$and $y^{\mu}\left(\sigma^{+}, \sigma^{-}\right)$with $\mu=1, \ldots, D-1$. Here $\sigma^{ \pm}=\frac{1}{2}\left(\tau_{ \pm}^{ \pm} \sigma\right)$, where $\tau$ and $\sigma$ are the natural time and spatial variables on the world sheet $\Sigma$. Then, the 2D $\sigma$-model action is given by

$$
S=\frac{1}{2} \int_{\Sigma} Q_{\mu \nu}^{+} \partial_{+} y^{\mu} \partial_{-} y^{\nu}-\partial_{+} y^{0} \partial_{-} y^{0}, \quad Q_{\mu \nu}^{+}=G_{\mu \nu}+B_{\mu \nu},
$$


where $G, B$ are the metric and antisymmetric tensor fields corresponding to the nontrivial part of the string background. The classical equations of motion are given by

$$
\begin{gathered}
\delta y^{0}: \quad \partial_{+} \partial_{-} y^{0}=0, \\
\delta y^{\mu}: \quad \partial_{+} \partial_{-} y^{\mu}+\left(\Gamma^{-}\right)_{\nu \lambda}^{\mu} \partial_{+} y^{\nu} \partial_{-} y^{\lambda}=0,
\end{gathered}
$$

where $\left(\Gamma^{ \pm}\right)_{\nu \lambda}^{\mu}=\Gamma_{\nu \lambda}^{\mu} \pm \frac{1}{2} H_{\nu \lambda}^{\mu}$ are the generalized connections that include the string torsion $H_{\mu \nu \lambda} \equiv \partial_{[\mu} B_{\nu \lambda]}$. We have implicitly imposed the conformal gauge in writing Eq. (2.14). Hence, the classical equations of motion are supplied with the constraints

$$
T_{ \pm \pm} \equiv \frac{1}{4} G_{\mu \nu} \partial_{ \pm} y^{\mu} \partial_{ \pm} y^{\nu}-\frac{1}{4} \partial_{ \pm} y^{0} \partial_{ \pm} y^{0}=0 .
$$

The conformal gauge allows for transformations $\sigma^{ \pm} \rightarrow f^{ \pm}\left(\sigma^{ \pm}\right)$, which can be used in a way consistent with the equations of motion (2.15), (2.16). We choose the socalled temporal gauge, where $y^{0}=\tau$. Then Eq. (2.15) is trivially satisfied whereas Eq. (2.16) remains unaffected since $G$ and $B$ are independent of $y^{0}$. The constraints (2.17) take the form

$$
G_{\mu \nu} \partial_{ \pm} y^{\mu} \partial_{ \pm} y^{\nu}=1 .
$$

For later use we define an angular variable $\theta$ via the relation

$$
G_{\mu \nu} \partial_{+} y^{\mu} \partial_{-} y^{\nu}=\cos \theta .
$$

The Euclidean signature of $K_{D-1}$ warrants the reality of $\theta$.

Clearly in the temporal gauge we may restrict our analysis entirely on $K_{D-1}$ and on the projection of the string world sheet $\Sigma$ on the $y^{0}=\tau$ hyperplane, following the spirit of the Lund-Regge analysis [1]. The resulting 2D surface $S$ has Euclidean signature with metric given by

$$
\begin{aligned}
d s^{2}= & G_{\mu \nu} d y^{\mu} d y^{\nu} \\
= & G_{\mu \nu}\left(\partial_{+} y^{\mu} \partial_{+} y^{\nu} d \sigma^{+2}+\partial_{-} y^{\mu} \partial_{-} y^{\nu} d \sigma^{-2}\right. \\
& \left.+2 \partial_{+} y^{\mu} \partial_{-} y^{\nu} d \sigma^{+} d \sigma^{-}\right) .
\end{aligned}
$$

Using the constraints (2.18) and the definition (2.19) we obtain from Eq. (2.20) the expression

$$
d s^{2}=d \sigma^{+2}+d \sigma^{-2}+2 \cos \theta d \sigma^{+} d \sigma^{-} .
$$

Thus, for $y^{0}=\tau$, determining the classical evolution of the string is equivalent to the problem of embedding the 2D surface $S$ with metric (2.21) on the $(D-1)$-dimensional space $K_{D-1}$. Hence the general analysis we have presented in the previous subsection becomes relevant to string theory at this point.

For further convenience we present the expressions for the nonvanishing Christoffel symbols and the Riemann curvature of the metric (2.21):

$$
\begin{gathered}
\Gamma_{ \pm \pm}^{ \pm}=\cot \theta \partial_{ \pm} \theta, \quad \Gamma_{\mp}^{ \pm}=-\frac{1}{\sin \theta} \partial_{\mp} \theta, \\
R_{+-+-}=-\sin \theta \partial_{+} \partial_{-} \theta .
\end{gathered}
$$

Contracting Eq. (2.16) with $G_{\mu \alpha} \xi_{\sigma}^{\alpha}$ where $\sigma=3, \ldots, D-1$, and using Eq. (2.4) we obtain

$$
\Omega_{+-}^{\sigma}=\Omega_{-+}^{\sigma}=\frac{1}{2} H_{\mu \nu \lambda} \xi_{\sigma}^{\mu} \partial_{+} y^{\nu} \partial_{-} y^{\lambda}, \quad \sigma=3, \ldots, D-1 .
$$

Contracting with $G_{\mu \alpha} \partial_{ \pm} y^{\alpha}$ and using Eq. (2.18) we obtain instead an identity and thus have no additional restrictions. Hence the information contained in the $D-1$ classical equations (2.16) is entirely encoded in the components of the second fundamental form (2.23) and in the two constraints (2.18). It will be convenient to modify the torsion $\mu_{ \pm}^{\sigma \tau}$ defined by Eq. (2.8), using a term that includes the string torsion for $i= \pm$ :

$$
\begin{aligned}
M_{ \pm}^{\sigma \tau} & \equiv \mu_{ \pm}^{\sigma \tau} \pm \frac{1}{2} H_{\mu \nu \lambda} \xi_{\sigma}^{\mu} \xi_{\tau}^{\nu} \partial_{ \pm} y^{\lambda} \\
& =G_{\mu \nu} \xi_{\sigma}^{\mu}\left(\partial_{ \pm} \xi_{\tau}^{\nu}+\left(\Gamma^{ \pm}\right)_{\lambda \alpha}^{\nu} \xi_{\tau}^{\lambda} \partial_{ \pm} y^{\alpha}\right) .
\end{aligned}
$$

It is evident that, similarly to $\mu_{ \pm}^{\sigma \tau}, M_{ \pm}^{\sigma \tau}$ is also antisymmetric, and thus nontrivial only for target spaces with dimension $D \geqslant 5$. After some tedious algebraic manipulations, Eqs. (2.11)-(2.13) for the remaining components of the second fundamental form $\Omega_{ \pm \pm}^{\sigma}$ and for the modified torsion $M_{ \pm}^{\sigma \tau}$ can be cast into the form

$$
\begin{gathered}
\Omega_{++}^{\tau} \Omega_{--}^{\tau}+\sin \theta \partial_{+} \partial_{-} \theta=-R_{\mu \nu \alpha \beta}^{+} \partial_{+} y^{\mu} \partial_{+} y^{\alpha} \partial_{-} y^{\nu} \partial_{-} y^{\beta} \\
\partial_{\mp} \Omega_{ \pm \pm}^{\sigma}-M_{\mp}^{\tau \sigma} \Omega_{ \pm \pm}^{\tau}-\frac{1}{\sin \theta} \partial_{ \pm} \theta \Omega_{\mp \mp}^{\sigma} \\
=R_{\mu \nu \alpha \beta}^{\mp} \partial_{ \pm} y^{\mu} \partial_{ \pm} y^{\alpha} \partial_{\mp} y^{\beta} \xi_{\sigma}^{\nu}
\end{gathered}
$$

and

$$
\begin{gathered}
\partial_{+} M_{-}^{\sigma \tau}-\partial_{-} M_{+}^{\sigma \tau}-M_{-}^{\rho[\sigma} M_{+}^{\tau] \rho}+\frac{\cos \theta}{\sin ^{2} \theta} \Omega_{++}^{[\sigma} \Omega_{--}^{\tau]} \\
=\left(R_{\mu \nu \alpha \beta}^{-}-D_{\mu}^{-} H_{\nu \alpha \beta}\right) \partial_{+} y^{\mu} \partial_{-} y^{\nu} \xi_{\sigma}^{\alpha} \xi_{\tau}^{\beta},
\end{gathered}
$$

where the curvatures are defined using the generalized connections $\left(\Gamma^{ \pm}\right)_{\nu \lambda}^{\mu}$,

$$
R_{\mu \nu \alpha}^{ \pm} \beta=-\partial_{[\mu}\left(\Gamma^{ \pm}\right)_{\nu] \alpha}^{\beta}+\left(\Gamma^{\mp}\right)_{\alpha[\mu}^{\gamma}\left(\Gamma^{ \pm}\right)_{\nu] \gamma}^{\beta},
$$

and similarly for the covariant derivatives $D_{\mu}^{-}$and $D_{\mu}^{+}$.

Next, counting the number of the embedding equations in Eqs. (2.25) $-(2.27)$ we find that there are $1+2(D-3)$ $+\frac{1}{2}(D-3)(D-4)$ of them, whereas the number of the unknown functions $\theta, \quad \Omega_{ \pm \pm}^{\sigma}, \quad$ and $M_{ \pm}^{\sigma \tau}$ is $1+2(D-3)+(D-3)(D-4)$. Hence, for $D \geqslant 5$ there are $\frac{1}{2}(D-3)(D-4)$ more unknown functions than equations. Notice, however, that the system (2.25)-(2.27) is invariant under local transformations on the world sheet generated by

$$
\begin{gathered}
\xi^{\mu} \rightarrow \Lambda^{-1} \xi^{\mu}, \quad \Omega_{ \pm \pm} \rightarrow \Lambda^{-1} \Omega_{ \pm \pm}, \\
M_{ \pm} \rightarrow \Lambda^{-1}\left(M_{ \pm}+\partial_{ \pm}\right) \Lambda,
\end{gathered}
$$


where $\Lambda=\Lambda\left(\sigma^{+}, \sigma^{-}\right)$is an orthogonal matrix of SO $(D-3)$. This gauge invariance accounts for the extra (gauge) degrees of freedom in Eqs. (2.25)-(2.27) and can be used to eliminate them (gauge fix).

\section{WZW backgrounds $K_{D-1}$}

It seems an enormous task to make further progress with the embedding system of Eqs. (2.25)-(2.27) as it stands in all generality. There are two major difficulties. First, the presence of sourcelike terms depending explicitly on $\partial_{ \pm} y^{\mu}$ and $\xi_{\sigma}^{\mu}$ seems to prohibit us from integrating them, even partially. Second, a Lagrangian description from which Eqs. (2.25)-(2.27) can be derived as equations of motion is also lacking.

It is rather remarkable that both problems can be solved by considering for $K_{D-1}$ either flat space or any WZW model based on a semisimple compact group $G$, with $\operatorname{dim}(G)=D-1$. This is due to the identities [11]

$$
R_{\mu \nu \alpha \beta}^{ \pm}=D_{\mu}^{ \pm} H_{\nu \alpha \beta}=0,
$$

which are generally valid for any WZW model. Then we completely get rid of the bothersome terms on the right-hand $\operatorname{side}^{2}$ of Eqs. (2.25)-(2.27). In order to show that a Lagrangian description exists, we first extend the range of definition of $\Omega_{++}^{\sigma}$ and $M_{+}^{\sigma \tau}$ by appending new components defined as

$$
\Omega_{++}^{2}=\partial_{+} \theta, \quad M_{+}^{\sigma 2}=\cot \theta \Omega_{++}^{\sigma}, \quad M_{-}^{\sigma 2}=-\frac{1}{\sin \theta} \Omega_{--}^{\sigma} .
$$

Then Eqs. (2.25)-(2.27) can be recast into the suggestive form

$$
\begin{gathered}
\partial_{-} \Omega_{++}^{a}+M_{-}^{a b} \Omega_{++}^{b}=0, \\
\partial_{+} M_{-}^{a b}-\partial_{-} M_{+}^{a b}+\left[M_{+}, M_{-}\right]^{a b}=0,
\end{gathered}
$$

where the new index $a=(2, \sigma)$. Notice that if we treat $\Omega_{++}^{a}$ not as a row of the bigger matrix $M_{+}^{a b}$ as suggested by Eq. (2.31), but as an independent vector, then the number of unknown functions in Eqs. (2.32) and (2.33) is augmented by $D-3$ compared to the same number of functions in Eqs. (2.25)-(2.27). However, there is a simultaneous enlargement of the local gauge symmetry from $\mathrm{SO}(D-3)$ to $\mathrm{SO}(D-2)$ that takes care of it. Such a gauge symmetry enlargement can only be achieved if the underlying change of variables is nonlocal. This will become more clear soon after the introduction of parafermions in the next section.

Equation (2.33) is a zero curvature condition for the matrices $M_{ \pm}$and it is solved (without worrying here about global issues related to the world sheet topology) by $M_{ \pm}=\Lambda^{-1} \partial_{ \pm} \Lambda$, where $\Lambda \in \mathrm{SO}(D-2)$. Then Eq. (2.32) can be written as

\footnotetext{
${ }^{2}$ Actually, the same result is obtained by demanding the weaker conditions $R_{\mu \nu \alpha \beta}^{-}-D_{\mu}^{-} H_{\nu \alpha \beta}=0$ and using the general identity $R_{\mu \nu \alpha \beta}^{-}-D_{\mu}^{-} H_{\nu \alpha \beta}=R_{\mu \nu \alpha \beta}^{+}-D_{\nu}^{+} H_{\mu \alpha \beta}$ and the property $R_{\mu \nu \alpha \beta}^{+}=R_{\alpha \beta \mu \nu}^{-}$. It would be interesting to find explicit examples where these weaker conditions hold.
}

$$
\partial_{-}\left(\Lambda^{a b} \Omega_{++}^{b}\right)=\partial_{-}\left(\Lambda^{a 2} \partial_{+} \theta+\partial_{+} \Lambda^{a 2} \tan \theta\right)=0 .
$$

The vector $\Lambda^{a 2}$ has unit length, i.e., $\Lambda^{a 2} \Lambda^{a 2}=1$. We can incorporate this constraint by defining $Y^{a}=\Lambda^{a 2} \sin \theta$. Then Eq. (2.34) assumes the form

$$
\partial_{-}\left(\frac{\partial_{+} Y^{a}}{\sqrt{1-Y^{2}}}\right)=0, \quad Y^{2} \equiv Y^{b} Y^{b}, \quad a, b=2,3, \ldots, D-1 .
$$

These equations were derived before in [2], while describing the dynamics of a free string propagating in $D$-dimensional flat space-time. It is remarkable that these equations remain unchanged even if the flat $(D-1)$-dimensional spacelike part is replaced by a curved background corresponding to a general WZW model. In retrospect, we may attribute this unexpected result to the fact that a group space is parallelizable, and it can be made flat in the sense of Eq. (2.30) with the addition of the appropriate amount of torsion. It should be emphasized that although the compatibility equations are universal, the actual evolution equations of the normal and tangent vectors to the surface are given by specializing Eqs. (2.9) and (2.10) to $K_{D-1}$; they are certainly different from those of the flat space free string.

As we have already mentioned, it would be advantageous if Eq. (2.35) (or an equivalent system) could be derived as classical equations of motion. The key that will enable us next to construct the corresponding Lagrangian is the observation that Eq. (2.35) imply chiral conservation laws, which are reminiscent of the equations obeyed by classical parafermions in coset models [12]. In fact Eq. (2.35) were derived as classical string equations for gauged WZW models corresponding to $\mathrm{SO}(D-1) / \mathrm{SO}(D-2)$ cosets in [4]; they are analytic continuations of the models $\mathrm{SO}(D-3,2) /$ $\mathrm{SO}(D-3,1)$ that give rise to string propagation in backgrounds with Lorentzian signature [13]. We mention for completeness that they also arise in the massless limit of the $\mathrm{SO}(D) / \mathrm{SO}(D-1)$ symmetric space sine-Gordon models, which were recently formulated as integrable perturbations of the $\mathrm{SO}(D-1) / \mathrm{SO}(D-2)$ gauged WZW models [9]. Since Eq. (2.35) themselves do not correspond to a Lagrangian system of equations, our strategy in the following will be to perform a nonlocal change of variables that maps them into Lagrangian form. This nonlocal change of variables is highly nonintuitive in differential geometry, and only the correspondence with parafermions makes it natural.

\section{DYNAMICS OF PHYSICAL DEGREES OF FREEDOM}

In this section we first briefly discuss some general aspects of gauged WZW models in connection with the associated coset conformal field theories. Then we restrict our attention to $\mathrm{SO}(D-1) / \mathrm{SO}(D-2)$ coset models and establish a relation between the chiral conservation laws obeyed by the corresponding parafermions and the embedding equations (2.35). At the end we present explicit results for $D=4$ and $D=5$. 


\section{Lagrangian description and parafermions}

Recall that the gauged WZW action is $[14,15]$

$$
\begin{aligned}
S= & I_{\mathrm{WZW}}(g)+\frac{k}{\pi} \int \operatorname{Tr}\left(A_{+} \partial_{-} g g^{-1}-g^{-1} \partial_{+} g A_{-}\right. \\
& \left.+A_{+} g A_{-} g^{-1}-A_{+} A_{-}\right),
\end{aligned}
$$

where $g \in G$ and $A_{ \pm}$are gauge fields valued in the Lie algebra of a subgroup $H \subset G$. The corresponding field strength is $F_{+-}=\partial_{+} A_{-}-\partial_{-} A_{+}-\left[A_{+}, A_{-}\right]$. We also split indices as $A=(a, \alpha)$, where $a \in H$ and $\alpha \in G / H$. Variation of Eq. (3.1) with respect to all fields gives the classical equations of motion

$$
\begin{gathered}
\delta A_{+}:\left.\quad D_{-} g g^{-1}\right|_{H}=0, \\
\delta A_{-}:\left.\quad g^{-1} D_{+} g\right|_{H}=0, \\
\delta g: \quad D_{-}\left(g^{-1} D_{+} g\right)+F_{+-}=0 .
\end{gathered}
$$

Imposing Eq. (3.3) on Eq. (3.4) yields the zero curvature condition $F_{+-}=0$ on shell, and

$$
\left.D_{-}\left(g^{-1} D_{+} g\right)\right|_{G / H}=0 .
$$

There are two commuting copies of an affine algebra corresponding to a WZW action for a group $G$, one for each chiral sector [16]. A remnant of this algebra is also present in the gauged WZW model. We parametrize the gauge fields as $A_{ \pm}=\left(\partial_{ \pm} h_{ \pm}\right) h_{ \pm}^{-1}$, where $h_{ \pm} \in H$. Thus, $h_{ \pm}$are given in terms of $A_{ \pm}$as

$$
h_{+}^{-1}=P e^{-\int^{\sigma^{+}} A_{+}}, \quad h_{-}^{-1}=P e^{-\int^{\sigma^{-}} A_{-}},
$$

where $P$ stands for path ordering. Using the gauge invariant group element

$$
f=h_{+}^{-1} g h_{+} \in G,
$$

and the on-shell zero curvature condition $F_{+-}=0$, we write Eq. (3.5) as

$$
\partial_{-} \Psi_{+}=0, \quad \Psi_{+}=\frac{i k}{\pi} f^{-1} \partial_{+} f \in G / H .
$$

Thus the coset valued matrix $\Psi_{+}$is chirally conserved.

In fact, $\Psi_{+}$are nothing but the classical parafermions [12]. Since they have Wilson lines attached to them [cf. Eqs. (3.7), (3.6)] they are nonlocal objects. This is also reflected in the algebra they obey [12] (we drop + as a subscript and denote $\sigma^{+}$by $x$ or $y$ )

$$
\begin{aligned}
\left\{\Psi_{\alpha}(x), \Psi_{\beta}(y)\right\}= & -\frac{k}{\pi} \delta_{\alpha \beta} \delta^{\prime}(x-y)-f_{\alpha \beta \gamma} \Psi_{\gamma}(y) \delta(x-y) \\
& -\frac{\pi}{2 k} f_{c \alpha \gamma} f_{c \beta \delta} \epsilon(x-y) \Psi_{\gamma}(x) \Psi_{\delta}(y),
\end{aligned}
$$

where the antisymmetric step function $\epsilon(x-y)$ equals $+1(-1)$ if $x>y(x<y)$. The last term in Eq. (3.9) is responsible for their nontrivial monodromy properties and un- usual statistics. In addition, conformal transformations are generated by $T_{++}=-\pi /(2 k) \Psi_{\alpha} \Psi_{\alpha}$.

The 2D $\sigma$ model having the above infinite dimensional symmetry is obtained by first choosing a unitary gauge by fixing $\operatorname{dim}(H)$ variables among the total number of $\operatorname{dim}(G)$ parameters of the group element $g$. Hence, there are $\operatorname{dim}(G / H)$ remaining variables, which will be denoted by $X^{\mu}$. Then, we eliminate the gauge fields in Eq. (3.1) using their equation of motion (3.2), (3.3),

$$
\begin{aligned}
& A_{+}^{a}=+i\left(C^{T}-I\right)_{a b}^{-1} L_{\mu}^{b} \partial_{+} X^{\mu}, \\
& A_{-}^{a}=-i(C-I)_{a b}^{-1} R_{\mu}^{b} \partial_{-} X^{\mu},
\end{aligned}
$$

where the appropriate shorthand definitions are

$$
\begin{gathered}
L_{\mu}^{a}=-i \operatorname{Tr}\left(t^{a} g^{-1} \partial_{\mu} g\right), \quad R_{\mu}^{a}=-i \operatorname{Tr}\left(t^{a} \partial_{\mu} g g^{-1}\right), \\
C^{a b}=\operatorname{Tr}\left(t^{a} g t^{b} g^{-1}\right) .
\end{gathered}
$$

Finally, the $\sigma$-model action is given by

$$
S=I_{\mathrm{WZW}}(g)-\frac{k}{\pi} \int_{\Sigma} L_{\mu}^{a}(C-I)_{a b}^{-1} R_{\nu}^{b} \partial_{+} X^{\mu} \partial_{-} X^{\nu} .
$$

\section{$\operatorname{SO}(D-1) / \mathrm{SO}(D-2)$ coset structure}

We specialize now to the $\mathrm{SO}(D-1) / \mathrm{SO}(D-2)$ gauged WZW model and show that Eq. (2.35) is equivalent to the parafermion equation (3.8). We will essentially follow the analysis of [4] adopted to our present purposes.

The group element $g \in \mathrm{SO}(D-1)$ in the right coset decomposition can be written as $g=\widetilde{h t}$, where

$$
\tilde{h}=\left(\begin{array}{cc}
1 & 0 \\
0 & h \in \mathrm{SO}(D-2)
\end{array}\right)
$$

and $t \in \mathrm{SO}(D-1) / \mathrm{SO}(D-2)$ is parametrized by a $(D-2)$-dimensional vector $\vec{X}$ as

$$
t=\left(\begin{array}{cc}
b & X^{j} \\
-X^{i} & \delta_{i j}-\frac{1}{b+1} X^{i} X^{j}
\end{array}\right), \quad b \equiv \sqrt{1-\vec{X}^{2}} .
$$

The range of the parameters $X^{i}$ is restricted by $\vec{X}^{2} \leqslant 1$ and the value of $b$ is such that the matrix $t$ is an element of SO $(D-1)$ obeying $t^{-1}=t^{T}$. Then we compute

$$
d t t^{-1}=\left(\begin{array}{cc}
0 & d X^{j}+\frac{\vec{X} \cdot d \vec{X}}{b(b+1)} X^{j} \\
-d X^{i}-\frac{\vec{X} \cdot d \vec{X}}{b(b+1)} X^{i} & \frac{1}{b+1} d X^{[i} X^{j]}
\end{array}\right)
$$




$$
t^{-1} d t=\left(\begin{array}{cc}
0 & d X^{j}+\frac{\vec{X} \cdot d \vec{X}}{b(b+1)} X^{j} \\
-d X^{i}-\frac{\vec{X} \cdot d \vec{X}}{b(b+1)} X^{i} & -\frac{1}{b+1} d X^{[i} X^{j]}
\end{array}\right) .
$$

To find an explicit expression for the parafermions in Eq. (3.8) we first rewrite the constraint (3.3) as

$$
\left(f^{-1} \partial_{+} f\right)_{i j}=\left(T^{-1} \partial_{+} T\right)_{i j}+\left(T^{-1} H^{-1} \partial_{+} H T\right)_{i j}=0,
$$

where $H=h_{+}^{-1} h h_{+}$and $T=h_{+}^{-1} t h_{+}$. The explicit form of $T$ is as in Eq. (3.14) with $X^{i} \rightarrow Y^{i} \equiv X^{j}\left(h_{+}\right)^{j i}$. Notice that since $\vec{Y}^{2}=\vec{X}^{2}$, the $Y^{i}$ are gauge invariant. Then we solve for

$$
\left(H^{-1} \partial_{+} H\right)_{i j}=\frac{1}{b(b+1)} \partial_{+} Y^{[i} Y^{j]} .
$$

The parafermion in Eq. (3.8) is computed by explicitly writing out $\Psi^{i} \equiv i k / \pi\left(f^{-1} \partial_{+} f\right)_{0 i}$ and utilizing Eq. (3.17). The final result is [4]

$$
\begin{gathered}
\Psi^{i}=\frac{i k}{\pi} \frac{\partial_{+} Y^{i}}{\sqrt{1-\vec{Y}^{2}}}=\frac{i k}{\pi} \frac{1}{\sqrt{1-\vec{X}^{2}}}\left(D_{+} X\right)^{j} h_{+}^{j i}, \\
\left(D_{+} X\right)^{j}=\partial_{+} X^{j}-A_{+}^{j k} X^{k} .
\end{gathered}
$$

Thus, the corresponding equation $\partial_{-} \Psi^{i}=0$ is precisely Eq. (2.35). The $Y^{i}$ are related to the $\sigma$-model variables nonlocally as

$$
Y^{i}=X^{j}\left(h_{+}\right)^{j i}, \quad h_{+}^{-1}=P e^{-\int^{\sigma^{+}} A_{+}},
$$

where the gauge field $A_{+}$is given by Eq. (3.10). This provides the necessary nonlocal change of variables that transform Eq. (2.35) into a Lagrangian system of equations.

The representation matrices for $\mathrm{SO}(D-1)$ are $\left(t_{A B}\right)_{C D}=\delta_{C[A} \delta_{B] D}$, where the indices split as $A=(0, i)$ with $i=1,2, \ldots, D-2$. Then the algebra of the parafermions (3.9) becomes

$$
\begin{aligned}
\left\{\Psi^{i}(x), \Psi^{j}(y)\right\}= & \frac{k}{2 \pi} \delta_{i j} \delta^{\prime}(x-y)-\frac{\pi}{2 k} \epsilon(x-y) \\
& \times\left(\delta_{i j} \Psi(x) \cdot \Psi(y)-\Psi_{j}(x) \Psi_{i}(y)\right) .
\end{aligned}
$$

The absence of linear terms in $\Psi^{i}$ on the right-hand side is due to the simple fact that $\mathrm{SO}(D-1) / \mathrm{SO}(D-2)$ is a symmetric space. Thus, structure constants involving only coset space indices are zero.

It remains to choose a gauge and explicitly compute $A_{+}$ and the $(D-2)$-component $\sigma$-model action (3.12). This has been done in another context for $\mathrm{SO}(3) / \mathrm{SO}(2)$ (the only Abelian case) in $[12,17]$, for $\mathrm{SO}(4) / \mathrm{SO}(3)$ in [4] and for $\mathrm{SO}(5)$ / $\mathrm{SO}(4)$ in [5]. Here, for the time being we proceed with a unified treatment of all $\mathrm{SO}(D-1) / \mathrm{SO}(D-2)$ models. It is convenient to distinguish between the cases of $D$ even or odd integers.

$D=2 N+2=$ even. We have enough gauge freedom to cast the orthogonal matrix $h \in \mathrm{SO}(2 N)$ and the vector $\vec{X}$ into the form

$$
h=\left(\begin{array}{cccccc}
\cos 2 \phi_{1} & \sin 2 \phi_{1} & 0 & \ldots & 0 & 0 \\
-\sin 2 \phi_{1} & \cos 2 \phi_{1} & 0 & \ldots & 0 & 0 \\
0 & 0 & 0 & \ldots & 0 & 0 \\
\vdots & \vdots & \vdots & & \vdots & \vdots \\
0 & 0 & 0 & \cdots & \cos 2 \phi_{N} & \sin 2 \phi_{N} \\
0 & 0 & 0 & \ldots & -\sin 2 \phi_{N} & \cos 2 \phi_{N}
\end{array}\right), \quad \vec{X}=\left(\begin{array}{c}
0 \\
X_{2} \\
0 \\
X_{4} \\
\vdots \\
0 \\
X_{2 N}
\end{array}\right)
$$

The total number of independent variables in $h$ and $\vec{X}$ is $2 N=D-2$, as it should be.

$D=2 N+3=o d d$. In such cases the orthogonal matrix $h \in \mathrm{SO}(2 N+1)$ and the vector $\vec{X}$ can be gauge fixed into the form

$$
h=\left(\begin{array}{ccccccc}
\cos 2 \phi_{1} & \sin 2 \phi_{1} & 0 & \ldots & 0 & 0 & 0 \\
-\sin 2 \phi_{1} & \cos 2 \phi_{1} & 0 & \ldots & 0 & 0 & 0 \\
0 & 0 & 0 & \ldots & 0 & 0 & 0 \\
\vdots & \vdots & \vdots & & \vdots & \vdots & \vdots \\
0 & 0 & 0 & \ldots & \cos 2 \phi_{N} & \sin 2 \phi_{N} & 0 \\
0 & 0 & 0 & \ldots & -\sin 2 \phi_{N} & \cos 2 \phi_{N} & 0 \\
0 & 0 & 0 & \ldots & 0 & 0 & 1
\end{array}\right), \quad \vec{X}=\left(\begin{array}{c}
0 \\
X_{2} \\
0 \\
X_{4} \\
\vdots \\
0 \\
X_{2 N} \\
X_{2 N+1}
\end{array}\right)
$$


Again the total number of the remaining independent variables is $2 N+1=D-2$, as it should be.

Using the above gauge fixing together with Eq. (3.15) and the Polyakov-Wiegman formula, we find that the WZW action (3.12) contributes to the total line element

$$
d s_{\mathrm{WZW}}^{2}=d \vec{\phi}^{2}+\frac{1}{2(1+b)} d \vec{X}^{2}+\frac{1+2 b}{4 b^{2}(1+b)^{2}}(\vec{X} \cdot d \vec{X})^{2},
$$

and has zero contribution to the total antisymmetric tensor. The contribution of the second term of Eq. (3.12) is more complicated and will not be presented here in all generality; of course, its effect will be taken into account in the specific examples below.

\section{Examples}

We will work out all the technical details in two examples. The first one is the Abelian coset $\mathrm{SO}(3) / \mathrm{SO}(2)$ [12]. In terms of our original problem it arises after solving the Virasoro constraints for strings propagating on 4D Minkowski space or on the direct product of the real line $R$ and the WZW model for SU(2), which is the only 3D non Abelian group for which a WZW action exists. The second example is the simplest nonAbelian coset based on $\mathrm{SO}(4)$ / $\mathrm{SO}(3)$ and was considered in [4]. In our context it arises in string propagation on 5D Minkowski space or on the direct product of the real line $R$ and the WZW model based on $\mathrm{SU}(2) \otimes \mathrm{U}(1)$.

$S O(3) / S O(2)$. Using Eq. (3.21) with $X_{2}=\sin 2 \theta$, we find that the solution for the gauge fields is

$$
A_{ \pm}=\left(\begin{array}{cc}
0 & 1 \\
-1 & 0
\end{array}\right)\left(1 \mp \cot ^{2} \theta\right) \partial_{ \pm} \phi
$$

and that the corresponding background has metric [12]

$$
d s^{2}=d \theta^{2}+\cot ^{2} \theta d \phi^{2} .
$$

Using Eq. (3.18), the corresponding Abelian parafermions $\Psi_{ \pm}=\Psi_{2} \pm i \Psi_{1}$ assume the familiar form

$$
\Psi_{ \pm}=\left(\partial_{+} \theta \pm i \cot \theta \partial_{+} \phi\right) e^{\mp i \phi \pm i \int \cot ^{2} \theta \partial_{+} \phi},
$$

up to an overall normalization.

The emergence of the $\mathrm{SO}(3) / \mathrm{SO}(2)$ parafermions can also be seen directly from the original system of embedding equations $(2.25)-(2.27)$. Since the indices $\sigma, \tau$ take only one value, the torsion matrix is $\mu_{ \pm}=0$. Then Eq. (2.27) is trivially satisfied, whereas Eqs. (2.25) and (2.26) give (after setting $\left.\Omega_{ \pm \pm}=\cot \theta / 2 \partial_{ \pm} \phi\right)$ the two equations

$$
\begin{gathered}
\partial_{+}\left(\cot ^{2} \frac{\theta}{2} \partial_{-} \phi\right)+\partial_{-}\left(\cot ^{2} \frac{\theta}{2} \partial_{+} \phi\right)=0, \\
\partial_{+} \partial_{-} \theta+\frac{\cos \frac{\theta}{2}}{2 \sin ^{3} \frac{\theta}{2}} \partial_{+} \phi \partial_{-} \phi=0 .
\end{gathered}
$$

These are the classical equations of motion of the $\mathrm{SO}(3)$ / $\mathrm{SO}(2)$ coset with metric (3.25) (up to rescaling of $\theta, \phi$ by a factor of 2) having the parafermions (3.26) as natural chiral objects. In the present geometrical context equations (3.27) were first derived in [1], whereas in [3] it was subsequently realized that they admit the $\mathrm{SO}(3) / \mathrm{SO}(2)$ coset interpretation we have just mentioned. It should be pointed out that for $D \geqslant 5$ a Lagrangian description for the embedding equations (2.25)-(2.27) cannot be possibly found in general without first making contact with parafermions, due to the fact that the torsion matrix $\mu_{ \pm}$(or $M_{ \pm}$) is nontrivial.

$S O(4) / S O(3)$. We parametrize $X_{2}=\sin 2 \theta \cos \omega$ and $X_{3}=\sin 2 \theta \sin \omega$ and use the basis of $\mathrm{SO}(3)$ representation matrices

$$
\begin{aligned}
t_{12}=\left(\begin{array}{ccc}
0 & 1 & 0 \\
-1 & 0 & 0 \\
0 & 0 & 0
\end{array}\right), \quad t_{13}=\left(\begin{array}{ccc}
0 & 0 & 1 \\
0 & 0 & 0 \\
-1 & 0 & 0
\end{array}\right), \\
t_{23}=\left(\begin{array}{ccc}
0 & 0 & 0 \\
0 & 0 & 1 \\
0 & -1 & 0
\end{array}\right) .
\end{aligned}
$$

Using Eq. (3.22) and the expansion for the gauge fields $A_{ \pm}=\sum_{i<j} A_{ \pm}^{i j} t_{i j}$ we find the solution

$$
\begin{aligned}
A_{+}^{12}= & -\left(\frac{\cos 2 \theta}{\sin ^{2} \theta \cos ^{2} \omega}+\tan ^{2} \omega \frac{\cos ^{2} \theta-\cos ^{2} \phi \cos 2 \theta}{\cos ^{2} \theta \sin ^{2} \phi}\right) \partial_{+} \phi \\
& -\cot \phi \tan \omega \tan ^{2} \theta \partial_{+} \omega, \\
A_{+}^{13}= & \tan \omega \frac{\cos ^{2} \theta-\cos ^{2} \phi \cos 2 \theta}{\cos ^{2} \theta \sin ^{2} \phi} \partial_{+} \phi+\cot \phi \tan ^{2} \theta \partial_{+} \omega,
\end{aligned}
$$

$$
A_{+}^{23}=\cot \phi \tan \omega \frac{\cos 2 \theta}{\cos ^{2} \theta} \partial_{+} \phi-\tan ^{2} \theta \partial_{+} \omega
$$

It turns out that an analogous expression for $A_{-}^{i j}$ can be found from Eq. (3.29) by writing all $\theta$ dependence in terms of $\cos 2 \theta$ and replacing $\cos 2 \theta$ by $1 / \cos 2 \theta$. Then, the background metric is [4]

$$
d s^{2}=d \theta^{2}+\tan ^{2} \theta(d \omega+\tan \omega \cot \phi d \phi)^{2}+\frac{\cot ^{2} \theta}{\cos ^{2} \omega} d \phi^{2},
$$

and the antisymmetric tensor is zero. The parafermions of the $\mathrm{SO}(4) / \mathrm{SO}(3)$ coset are non-Abelian given by Eq. (3.18) with covariant derivatives (omitting an overall factor of 2 )

$$
\begin{gathered}
\frac{\left(D_{+} X\right)^{1}}{\sqrt{1-\vec{X}^{2}}}=\frac{\cot \theta}{\cos \omega} \partial_{+} \phi, \\
\frac{\left(D_{+} X\right)^{2} \pm i\left(D_{+} X\right)^{3}}{\sqrt{1-\vec{X}^{2}}}=e^{ \pm i \omega}\left( \pm i \tan \theta\left(\tan \omega \cot \phi \partial_{+} \phi+\partial_{+} \omega\right)\right. \\
\left.+\partial_{+} \theta\right) .
\end{gathered}
$$


As a check, one may verify that $\Psi^{i} \Psi^{i}$ $=1 /\left(1-\vec{X}^{2}\right)\left(D_{+} X\right)^{i}\left(D_{+} X\right)^{i}$ is indeed proportional to the $T_{++}$component of the energy momentum tensor corresponding to a $\sigma$ model with metric (3.30).

In addition to the two examples above, there also exist explicit results for the coset $\mathrm{SO}(5) / \mathrm{SO}(4)$ [5]. This would correspond in our context to string propagation on a $6 \mathrm{D}$ Minkowski space or on the background $R$ times the SU(2) $\otimes \mathrm{U}(1)^{2} \mathrm{WZW}$ model. It should be pointed out that there is no reason to demand conformal invariance for the backgrounds with metrics (3.25) and (3.30) because they arise in a different context describing the geometry of the physical degrees of freedom.

\section{CONCLUSIONS}

We have investigated some universal aspects of classical string dynamics by integrating the Gauss-Codazzi equations of the corresponding embedding problem. We found for the class of $D$-dimensional backgrounds $R \otimes K_{D-1}$, where $K_{D-1}$ is $R^{D-1}$ or the WZW model for a general $(D-1)$ dimensional semisimple compact group, that there are $D-2$ physical degrees of freedom whose dynamics is governed by the $\mathrm{SO}(D-1) / \mathrm{SO}(D-2)$ coset conformal field theory. The parafermion variables of this coset arise naturally in the present geometrical context, and so our results could be viewed as a link between conformal field theory techniques and the classical differential geometry of embedding surfaces.

There are two obvious extensions one can further make. First, suppose we start with a $D$-dimensional string background with signature $(2, D-2)$. The "spatial" part of this background is now Lorentzian, and therefore one has to consider suitable analytic continuation of the previous results. In particular, instead of the coset $\mathrm{SO}(D-1) / \mathrm{SO}(D-2)$ we find that the dynamics of the physical degrees of freedom is now given by the noncompact coset $\mathrm{SO}(D-3,2) /$ $\mathrm{SO}(D-3,1)$. The simplest version of this for $D=4$ has already been considered in [18]. Second, it is also interesting to consider various supersymmetric generalizations of the present framework.

There are many similarities between classical string dynamics and the theory of ordinary 2D $\sigma$ models. The latter can also be viewed as describing the embedding of $2 \mathrm{D}$ sur- faces into a group or coset space manifold, which in turn is embedded in flat space. Exploiting classical conformal invariance, which is similar to choosing the orthonormal gauge in string theory, amounts to reducing ordinary $\sigma$ models to the so-called symmetric space sine-Gordon models (SSG) [6-8]. The SSG models have been described as perturbations of conformal field theory cosets [9]; for example, the reduced $S^{n}=\mathrm{SO}(n+1) / \mathrm{SO}(n) \sigma$ model yields an integrable sineGordon perturbation of the $\mathrm{SO}(n) / \mathrm{SO}(n-1)$ coset conformal field theory. Hence, apart from the potential terms, and in the absence of string self-interactions, the structure of the kinetic terms is the same for the two classes of embedding problems. It is interesting to note that other reduced $\sigma$ models for general symmetric spaces have been described using appropriately chosen gauged WZW cosets (plus perturbations). Therefore, the parafermion variables of the corresponding coset conformal field theories (at and away from the conformal point) also play a key role in the integration of the embedding equations.

Finally, an interesting issue is the quantization of string theory. There are two different methods of quantizing constrained systems, either by solving the classical constraints and then quantize directly the physical degrees of freedom, or by quantizing the unconstrained degrees of freedom and then impose the constraints as quantum conditions on the physical states. It is well known that in general these two methods of quantization are not equivalent, in particular when the constraints have quadratic form as in string theory. Quantization of string theory usually proceeds using the second method, but in the present framework the physical degrees of freedom should be quantized directly using the quantization of the associated parafermions. Exploring this issue further is an interesting problem.

\section{ACKNOWLEDGMENTS}

One of us (I.B.) wishes to thank the Institute for Theoretical Physics in Utrecht for hospitality and financial support during the last stages of this research. The work of K.S. was carried out with the financial support of the European Union Research Program "Training and Mobility of Researchers", and is part of the project "String Duality Symmetries and Theories with Space-Time Supersymmetry,', under Contract No. ERBFMBICT950362.
[1] F. Lund and T. Regge, Phys. Rev. D 14, 1524 (1976).

[2] B. Barbashov, V. Nesterenko, and A. Chervyakov, Theor. Math. Phys. 59, 458 (1984).

[3] I. Bakas, Phys. Lett. B 319, 457 (1993); Int. J. Mod. Phys. A 9, 3443 (1994).

[4] I. Bars and K. Sfetsos, Mod. Phys. Lett. A 7, 1091 (1992); Phys. Rev. D 46, 4495 (1992).

[5] I. Bars and K. Sfetsos, Phys. Lett. B 277, 269 (1992); Phys. Rev. D 46, 4510 (1992).

[6] K. Pohlmeyer, Commun. Math. Phys. 46, 207 (1976); K. Pohlmeyer and K. Rehren, J. Math. Phys. 20, 2628 (1979); H. Eichenherr and J. Honerkamp, ibid. 22, 374 (1981).

[7] F. Lund, Phys. Rev. Lett. 38, 1175 (1977).
[8] R. D’Auria, T. Regge, and S. Sciuto, Nucl. Phys. B171, 167 (1980).

[9] I. Bakas, Q-Han Park, and H.-J. Shin, Phys. Lett. B 372, 45 (1996).

[10] L. Eisenhart, Riemannian Geometry (Princeton University Press, Princeton, NJ, 1964).

[11] T.L. Curtright and C.K. Zachos, Phys. Rev. Lett. 53, 1799 (1984); E. Braaten, T.L. Curtright, and C.K. Zachos, Nucl. Phys. B260, 630 (1985).

[12] K. Bardakci, M. Crescimanno, and E. Rabinovici, Nucl. Phys. B344, 344 (1990); K. Bardakci, M. Crescimanno, and S.A. Hotes, ibid. B349, 439 (1991). 
[13] I. Bars and D. Nemeschansky, Nucl. Phys. B348, 89 (1991).

[14] D. Karabali, Q-Han Park, H.J. Schnitzer, and Z. Yang, Phys. Lett. B 216, 307 (1989); D. Karabali and H.J. Schnitzer, Nucl. Phys. B329, 649 (1990).

[15] E. Witten, Nucl. Phys. B223, 422 (1983); K. Bardakci, E. Rabinovici, and B. Säring, ibid. B299, 151 (1988); K.
Gawedzki and A. Kupiainen, Phys. Lett. B 215, 119 (1988); Nucl. Phys. B320, 625 (1989).

[16] E. Witten, Commun. Math. Phys. 92, 455 (1984).

[17] E. Witten, Phys. Rev. D 44, 314 (1991).

[18] H. de Vega, J. Mittelbrunn, M. Medrano, and N. Sanchez, Phys. Lett. B 323, 133 (1994). 